\title{
How Well Can Architecture Students Comprehend the Site as Design Context without Performing On-site Observation?
}

\author{
Aldyfra Luhulima Lukman' ${ }^{1}$, Sahid $^{1}$ \\ ${ }^{1}$ Architecture Department, Parahyangan Catholic University, Bandung Indonesia \\ aldyfra@unpar.ac.id
}

\begin{abstract}
The Covid-19 pandemic has forced daily activities, including educational activities, to prevent or minimize the transmission or spread of the disease caused by a coronavirus. Online architecture education demands significant adjustments in the embedded learning methods, especially those that entail architecture design studio. An architecture design studio that commonly involves direct interaction in physical spaces should be performed through online media and computer screens. Architecture students' comprehension of the site or design contexts is usually formed by collecting information through on-site observation. In an online architecture design studio, an on-site survey is replaced by observing visual representation of the site. This study aims to identify how well architecture students in architecture design studios can comprehend the site when they rely on data acquired from virtual observation and on secondary data and how beneficial the comprehension of their design process is. Students' understanding of the site was measured with thirteen site attributes. It can be concluded that when architecture students depend on mere audio and visual data collected from the internet and other people, without visiting the site, they tend to have partial comprehension regarding the site as context. Contextual and responsive design demands a comprehensive understanding of all site attributes. The architecture design studio should incorporate on-site observation concerning the site according to the health protocol so that the architecture students involved in the studio can better understand the site and create more contextual and responsive designs.
\end{abstract}

CC 2021 IJBESR. All rights reserved.

Keywords: Architecture Students, Design Context, Online Architecture Design Studio, On-Site Observation, Site Comprehension, Virtual Observation

\section{Introduction}

The Covid-19 pandemic has forced daily activities to be adjusted to prevent or minimize the transmission or spread of the disease caused by a coronavirus. Several steps have been implemented consistently and legalized in policy and protocol, commonly called health protocol, for dealing with the pandemic. Health protocol encompasses:

1. Maintaining personal and environmental hygiene.

2. Wearing masks when performing outof-home activities.

3. Staying away from the crowds.
4. Keeping physical and social distance from other people when performing out-of-home activities.

5. Reducing mobility.

Health policy and protocol have compelled most out-of-home activities (including educational activities) to be conducted in or from home.

Educational activities during this pandemic era (including in Indonesia), from an early age to higher education, are carried out using online media. Online learning that previously was considered secondary or optional in higher education, including in architecture education, has been regarded as primary or compulsory in 
the era of the Covid-19 pandemic. Online architecture education demands significant adjustments in the embedded learning methods, especially those that entail architecture design studio. In the pandemic era, an architecture design studio that commonly involved direct interaction in physical spaces between educators and students should be performed through online media and computer screens. Similar to other ways of learning and working facing disruptions due to the Covid-19 pandemic, the implementation of an online architecture design studio must deal with issues in terms of the learning process and outputs.

Several studies have addressed issues in online architecture design studios. Some previous studies highlighted the effectiveness of methods and processes of teaching and learning in online architecture design studios [1,2,3]. Other studies emphasized the potential and challenges of implementing an online architecture design studio related to learning outputs [4,5]. This study embraces a few materials of previous studies in terms of learning process and outputs but chooses to focus on a more initial and substantial aspect of architecture design studio, which is students' comprehension of design context, specifically the site. Understanding the context, including location, determines how architects respond to potential problems related to the context in their design. The referenced studies have not specifically discussed the issue regarding context or site comprehension in the architecture design studio.

Similar to other fields in higher education, studios and other core courses in architecture education that has been implemented from the era of Ecole des Beaux-Arts to the era of a preCovid-19 pandemic, which commonly requires interactions between educators and students in a physical setting [2], must shift their paradigms and methods during the time of Covid-19 pandemic. The virtual or online studio has advantages and drawbacks compared to a physical or offline studio. The common problems of the online studio are more related to the technology, psychological aspect, social interaction, and skill development [2, 3, 4].

Architecture design studio serves as the center of architecture education by providing a setting wherein knowledge originating from different fields is integrated through intensive training to develop design skills [1,4]. Students in an architecture design studio handle a design project by constructing solutions to a design problem through discussion with lecturers/ mentors/ instructors [1]. Architecture design studio applies "learning by doing" approaches and provides an experience that enables students to understand various ways to develop creativity and design [4]. Assessment and marking in architecture design studio encompass products and processes [1]. The architecture design studio is regarded as an environment having structure and culture that shapes and supports lifelong learner characteristics such as independence, critical thinking, and reflective analysis [1]. The architecture design studio also possesses a social role by providing the social interaction of students, lecturers/ mentors/ instructors, and juries/ reviewers.

The cultural and structural differences between the architecture design studio and typical classes are marked by the development of knowledge and skills (theoretical, technical, and social) containing tangible and intangible aspects [4]. The learning process in an architecture design studio reflects architectural practices performed by a professional architect in the real world. In an architecture design studio, students enter a simulated environment with all its constraints and rules, which is shaped and determined by the lecturer/ mentor/ instructor, and then learn how to design through different forms of discussion consisting of an individual tutorial, group mentoring, and crit $[1,4]$. Students' works are constantly assessed by lecturers/ mentors/ instructors spending a 
specific period reviewing each student's work individually. Eventually, external examiners, experts, practitioners, or other students can review students' results in the crit session [1]. The parties in conventional crit usually gather in a physical space, whereas those involved in virtual crit sessions use a digital platform [4]. In actual architectural practices, the role played by students reflects the role of professional architects, whereas the critics represent the role of investors, policymakers, project owners, and users.

The actual practices simulated in the architecture design studio are expected to prepare the students for taking an initial step into the world of architects. Students who choose not to work as professional architects in the future can still take advantage of design thinking, skills learned, and characteristics shaped in the architecture design studio to excel in their selected field. Benefits obtained by students in physical architecture design studios are expected to be maintained in the virtual or online architecture design studio. Several studies have addressed issues in online architecture design studios. Some previous studies highlighted the effectiveness of methods and processes of teaching and learning in online architecture design studios [1,2,3]. Other studies emphasized the potential and challenges of implementing an online architecture design studio related to learning outputs [4,5]. This study embraces a few materials of previous studies in terms of learning process and outputs but chooses to focus on a more initial and substantial aspect of architecture design studio, which is students' comprehension of design context, specifically the site. Understanding the context, including location, determines how architects respond to potential problems related to the context in their design. The referenced studies have not specifically discussed the issue regarding context or site comprehension in the architecture design studio.
In terms of preparing students for professional practices after graduating, the virtual or online studio gives the opportunity to enhance students' independence, responsibility, and research skill, which eventually also strengthens multidisciplinary characteristics in architectural pedagogy [1,3]. "Learning by doing" and the reflective process can still be involved in the virtual studio [3]. However, the virtual studio is deemed more beneficial to final-year students than to first-year students who still need a lot of direct interactions to develop their abilities in abstraction, conception, spatial thinking, and coordination between brain and hands, such as sketching and model-making $[3,4,6]$.

In terms of the time and methods of delivering learning materials, the online studio can be conducted in synchronous (real-time) or asynchronous mode, depending on the needs and availabilities of students [4]. The online studio provides an opportunity to acquire materials according to students' availability through discussion or presentation recordings [1]. Taking perspectives regarding expenses and access to the learning materials into account, an online studio is regarded as more economical and provides more flexibility than a physical studio [1].

The architecture design studio can serve as a setting for discussion, presentation, participation, and education [4]. Social activities can still occur in an online studio, although their media, ways, and behaviors are different from those in a physical studio [4]. The social component of a physical studio considered missing in an online studio is the bonding formed by informal, unstructured, unplanned activities involving students' interactions $[3,4]$. This bonding seems to be regarded by the students as an important social part of the architecture design studio.

How students respond to the implementation of online architecture design studio and the 
rationales behind their responses have served as the focus of discussing architecture education during the era of the Covid-19 pandemic. Previous studies measuring students' reactions to the implementation of online architecture design studio concluded that the students prefer hybrid format combining online and offline learning methods during and after the era of the Covid-19 pandemic to entirely online mode [3, 4]. The offline activities expected to be incorporated in the hybrid format of architecture design studios are regarded as having more impact on students' design skills development. A survey conducted by Salama and Crosbie [5] in the United Kingdom displays that during the implementation of an online architecture design studio, there were issues regarding students' satisfaction, interaction, sense of community, motivational supports, mental health problems, and increasing gap between architecture education and practice. The results of this survey might not be generalized; however, they can be used to form a basis for determining the mode of architecture studio post-Covid-19 pandemic [5]. Selecting the mode of architecture design studio needs to consider which design components can involve entirely online methods and which design components still require offline approaches.

Context is a vital component of an architecture design. Architecture, which covers outdoor and indoor spaces, should fit the context(s) [7]. Responses to the contexts require comprehension of the contexts. Reading and comprehending the site as context are fundamental components in the architectural design process [8]. How the architect relates themself to the site determines their architectural proposal and outputs [8]. Comprehending sites, especially in the early phases of architectural design studio, is still deemed an issue that should be anticipated in the virtual or online implementation, which has not been sufficiently addressed in previous studies concerning online architecture design studios. Spatial comprehension derived from bodily experience in the actual site would be different from the understanding attained from virtual observation.

Students' comprehension regarding site or contexts is typically formed by collecting information through on-site observation [4]. In an online architecture design studio, on-site observation commonly embraces various senses, is replaced by observing visual representation of the site, such as photos, videos, or digital records of the site, which depends mainly on mere visual sense [4]. Visual perception of space, whether it entails on-site or online observation, relies on these components: colors, light, texture, size, form, movement, rhythm, and relationship among these components $[9,10]$. Perception of space can affect the observer emotionally. Affective or subjective aspects of perception entailed in an on-site observation such as mood, emotion, feelings, preference, attitudes, imagination, memory, or thought [10] might differ from virtual surveys involving photos and videos. Architecture students are trained to "read" and comprehend the site through bodily experience; their bodies move and navigate in the site. They have a more substantial sensory relationship with the site than the connection made from virtual observation. Physical and psychological responses related to the visual sense of architecture students as observers have a wide variety of forms when dealing with different modes of representation $[10,11]$.

Comprehending site as design context is positioned as the focus of this study by assuming that differences between the spatial perception of observers who perform on-site surveys and those who conduct online surveys lead to different levels of site comprehension. Site comprehension of architecture students is measured by referring to site attributes proposed by LaGro Jr. [12] that include: 1) Physical attributes; 2) Biological attributes; 3) Cultural Attributes. Physical attributes consist of the shape and size of the site, topography, 
geology, hydrology, soils, climate, and natural hazards. Biological attributes encompass ecological communities, vegetation, and wildlife. Cultural attributes comprise land use and tenure, regulations, property value, public infrastructure, building and neighborhood characteristics, historic resources, and sensory perception. This study aims to identify how well architecture students in architecture design studios can comprehend the site when they must rely on data acquired from virtual observation and on secondary data and how beneficial the comprehension to their design process is.

\section{Material and Methods}

This qualitative-evaluative study uses a historical approach and consists of the following stages: 1) Literature review, 2) Questionnaire formulation, 3) Data Collection, 4) Data input and processing, 5) Data Analysis, 6) Synthesis and Conclusion. The qualitative and quantitative data were collected through Google Form questionnaires distributed to respondents through email or other social media. The questions in the questionnaires incited students to recall their memories and experiences when performing virtual observation as a part of an online architecture design studio. Questionnaire materials referred to site attributes proposed by LaGro Jr. [12]; a few attributes were adjusted and/or combined for the questions' familiarity, conciseness, and comprehensiveness. The questions contain 13 characteristics: 1) site shape and boundaries; 2) topography; 3) hydrology; 4) soils; 5) climate; 6) hazards; 7) vegetations and animals; 8) Land use, tenure, property value, or property value; 9) Infrastructure; 10) Building characteristics; 11) Neighborhood characteristics; 12) Historic resources; 13) Sensory perception. Data were inputted and processed in Microsoft Excel. Synthesis and conclusion were formulated qualitatively.
Time constraint was the primary factor in deciding which architecture students would be involved in this study. It was decided that respondents of this study are second-year architecture students in the Undergraduate Program, Department of Architecture, Parahyangan Catholic University, Bandung, West Java, Indonesia, who had passed online Architecture Design Studio 4 in the second semester of the academic year 2020/2021. The decision was based on these considerations:

1. Architecture Design Studio 4 in the second semester of the academic year 2020/2021 was held in an entirely online format for the whole semester.

2. Some students in this Architecture Design Studio who were unable to perform on-site observation due to restricted mobility in the era of the Covid-19 pandemic had to rely on digital media for collecting data of the site.

3. Comprehension concerning the site and the surrounding area has been considered vital design contexts in the assigned project in this Architecture Design Studio, which is the design of a multifunction complex (with the hotel as the primary function) in North Bandung, West Java, Indonesia.

4. Second-year students involved in this Architecture Design Studio were assumed to have basic architectural knowledge and skills already but still require a lot of offline interactions to develop their comprehension of the site and other contexts.

\section{Results and Discussions}

There were 137 second-year architecture students in the Undergraduate Program, Department of Architecture, Parahyangan Catholic University, Bandung, West Java, Indonesia, who had passed online Architecture Design Studio 4 in the second semester of the academic year 2020/2021 and been sent the Google Form Questionnaires. One hundred thirteen students filled the questionnaires. There are 11 invalid data out of 113 responses, so that there are 102 valid responses included in 
the analysis. The profiles of respondents who provided accurate data are described below:

- There are 97 respondents classified as batch 2019/ second-year students (95\%) and 5 (five) respondents classified as batch 2018/ third-year students (5\%) who provided valid data (see Figure 1).

- Respondents providing valid data consist of 61 females (60\%), and 41 males $(40 \%)$ provide accurate data (see Figure 2).

- There are 61 respondents (60\%) not performing on-site observation at all and 41 respondents $(40 \%)$ performing on-site observation at least once who provided valid data (see Figure 3).

Seven respondents $(7 \%)$ conducted mere onsite observation. Seven respondents (7\%) collected secondary data from other students, lecturers/mentors, or experts without performing on-site observation at all. Thirtyfour respondents (33\%) completed on-site observation combined with collecting data from the internet or other students, lecturers, or experts. Fifty-four respondents $(53 \%)$ collected data from the internet and other students, lecturers or experts, without performing on-site observation at all (see Figure 4).

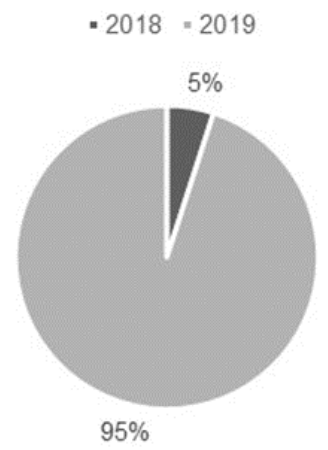

Source: Lukman and Sahid, 2021

Figure 1: Batch of the respondents

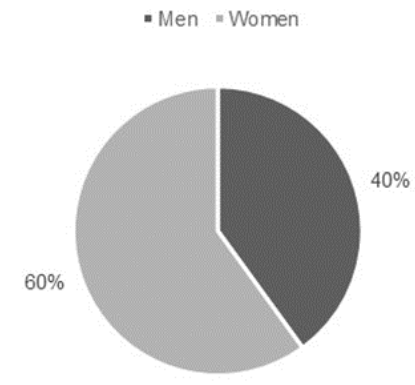

Figure 2: Gender of the respondents Source: Lukman and Sahid, 2021

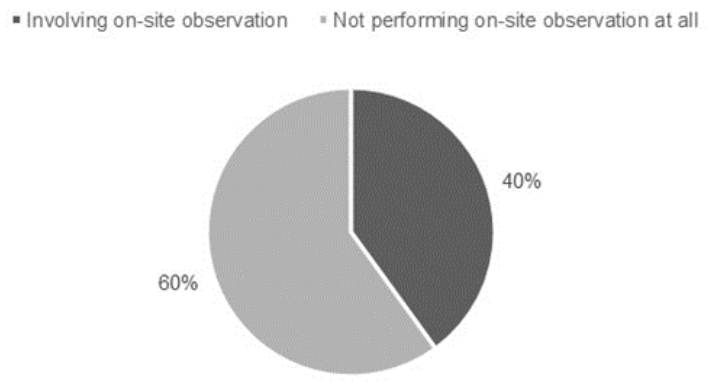

Source: Lukman and Sahid, 2021

Figure 3: The incorporation of on-site observation in a survey conducted by the respondents

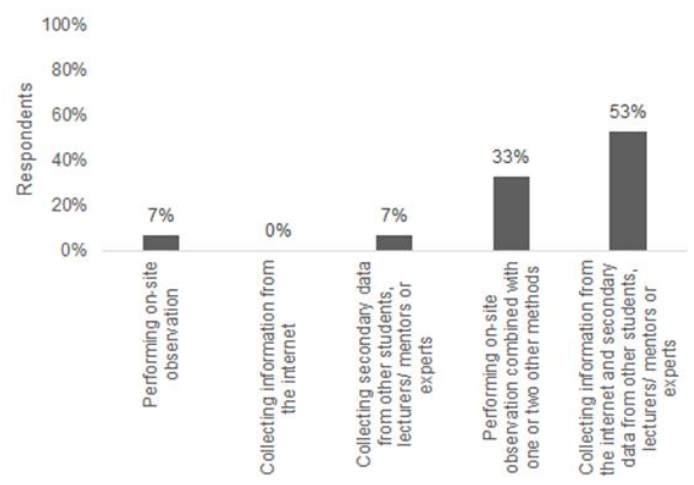

Source: Lukman and Sahid, 2021

Figure 4: Methods of data collection that were conducted by the respondents in site observation

Whether they performed on-site observation or not, respondents were asked to inform the rationale(s) of their selected method(s) for collecting the data. Respondents who conducted on-site observation at least once gave the following rationales (see Figure 5): 1) 
Difficulties of comprehending the site digitally $(56 \%), 2)$ Expectation concerning acquiring essential information from the site $(86 \%), 3)$ Expectation regarding collecting more nonphysical data from the site $(73 \%), 4)$ Issue related to the accuracy of online data (2\%), and $5)$ Easy access to the site (2\%). Respondents who did not carry out on-site observation at all informed these following rationales (see Figure $6): 1)$ Students did not live in the city where the site is located $(89 \%)$, 2) Concern about the possibility of contracting Covid-19 (39\%), 3) Health condition that did not enable the students to perform on-site observation $(5 \%), 4)$ On-site observation had been delegated to other students in the team (30\%), and 5) Data from the internet were regarded sufficient to complement the information regarding the site $(8 \%)$. The data indicate that acquiring actual and comprehensive data was the primary motivation for performing on-site observation. In contrast, an issue concerning the possibility of contracting the Covid-19 and the site's location in terms of distance and access were the prime factors causing the students to avoid on-site observation.

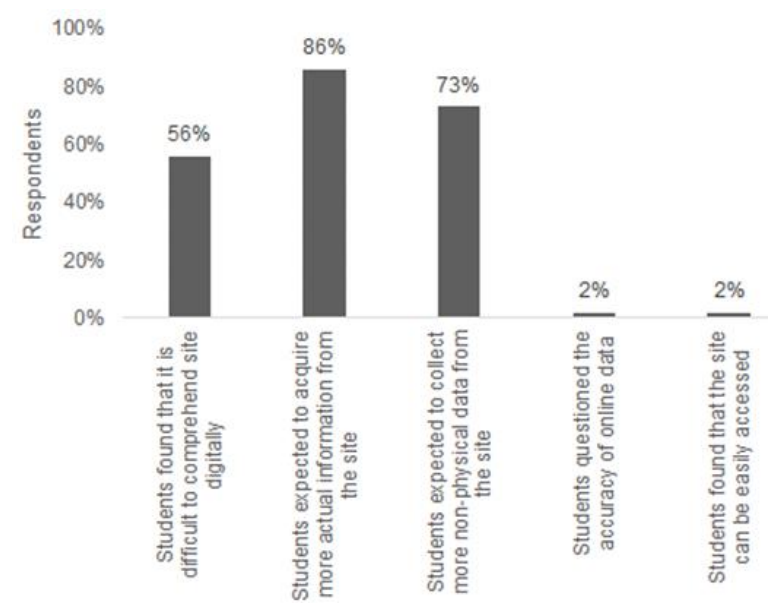

Source: Lukman and Sahid, 2021

Figure 5: Rationale of performing on-site observation

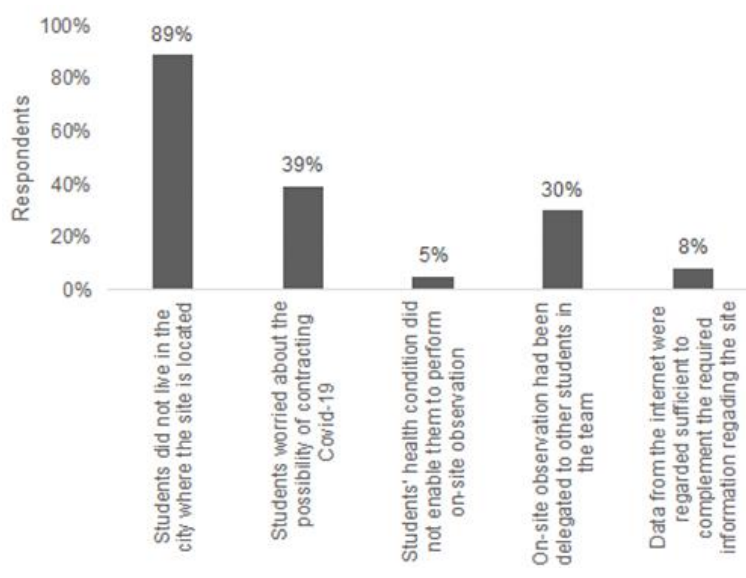

Source: Lukman and Sahid, 2021

Figure 6: Rationale of not performing on-site observation

The further analysis only addresses 61 respondents who did not perform on-site observation at all. These 61 respondents were being asked whether the information regarding the site was sufficient for comprehending the site when they depended on the internet or other people as the sources. The adequacy of information regarding the site was measured in relation to 13 attributes referring to LaGro Jr. (2008).

There are six attributes of the site regarded as being identifiable by most of the respondents who did not perform on-site observation at all, by referring to data collected from the internet and/or from other people assumed as having more knowledge and understandings regarding the site such as lecturers/mentors, experts or other students. These six attributes consist of (see Figure 7): 1) Site shape and boundaries (which can be identified by $64 \%$ respondents); 2) Climate (which can be identified by $87 \%$ respondents); 3) Land use, tenure, regulation and property value (which can be identified by $77 \%$ respondents); 4) Infrastructure (which can be identified by $58 \%$ respondents); 5) Building characteristics (which can be identified by $72 \%$ respondents); 6) Historic resources (which can be identified by $59 \%$ respondents). The other 
seven attributes of the site are deemed as less/ not identifiable by most of the respondents who did not perform on-site observation at all when collecting the information depended on data collected from the internet and/or from other people assumed as having more knowledge and understandings regarding the site such as lecturers/mentors, experts, or other students.

These seven attributes comprise (see Figure 7): 1) Topography (which can be identified by only $48 \%$ respondents); 2) Hydrology (which can be identified by only $41 \%$ respondents); 3) Soils (which can be identified by only $12 \%$ respondents); 4) Hazards (which can be identified by only $35 \%$ respondents); 5) Vegetations and animals (which can be identified by only $48 \%$ respondents); 6) Neighborhood characteristics (which can be identified by only $24 \%$ respondents); 7) Sensory perception (which can be identified by only $37 \%$ respondents).

Based on the data, it can be defined that collecting information of some site attributes does not require whole bodily experience. Respondents could obtain information concerning and understand the attributes of the site by depending on the mere visual sense, such as six attributes that were easily identified by those who rely on audio and visual materials and did not perform on-site observation at all, which consist of 1) Site shape and boundaries; 2) Climate; 3) Land use, tenure, regulation, and property value; 4) Infrastructure; 5) Building characteristics; 6) Historical resources. These six attributes can be understood through observing primary or secondary data in the form of photos, videos, verbal statements, and/ or writings. The other seven attributes that were not easily identified by those who did not perform on-site observation at all are assumed as requiring full bodily experience, which involves various senses (visual, auditory, olfactory, haptic) for comprehending the site. It can be stated that visual or audio information acquired through photos, videos, verbal statements, and writings is regarded inadequate for understanding these seven attributes, which consist of 1) Topography; 2) Hydrology; 3) Soils; 4) Hazards; 5) Vegetations and animals; 6) Neighborhood characteristics; 7) Sensory perception.

Whether they were adequately identifiable or not, attributes relate to each other. The connection between climate and sensory perception can be presented as an example of the relationship. Information about the degree of temperature around or in the site can easily be obtained from the internet or other people having more knowledge about the site. Nevertheless, determining whether that degree of temperature around and in the site is deemed as hot or cold might require an on-site survey. Signifying the sensation or feeling of "hotness" or "coolness" needs the presence of the observer on the site rather than mere visual observation.

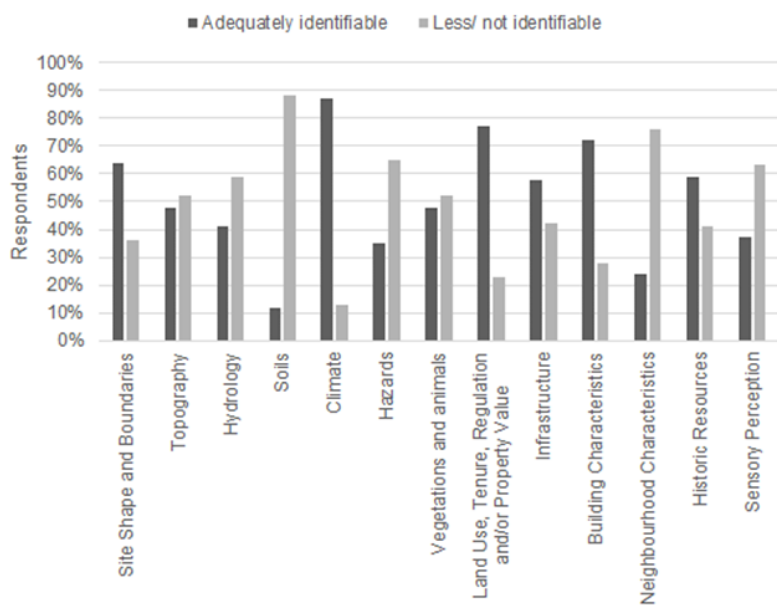

Source: Lukman and Sahid, 2021

Figure 7: Level of identification of the site attributes

When a respondent not performing on-site observation at all answered that they could not identify a specific attribute of the site adequately by depending on online or secondary data only, the respondent was asked to select the method(s) that was/were assumed as suitable for improving and/or 
complementing the information regarding that specific attribute. Respondents not performing on-site observation suggested that asking for secondary data from lecturers/ mentors/ instructors, experts, or other students is considered the best method for improving and complementing the information regarding the site attribute, which entails 11 of 13 attributes (see figure 8). Respondents who valued asking for secondary data from lecturers/ mentors/ instructors, experts, or other students as the best method for improving and complementing the information regarding the site attributes have a percentage range of $77-88 \%$ for these 11 attributes (see figure 8). These 11 attributes are: 1) Site shape and boundaries, 2) Topography, 3) Hydrology, 4) Soils, 5) Hazards, 6) Vegetations and animals, 7) Land use, tenure, regulation, and property value, 8) Infrastructure, 9) Building characteristics, 10) Neighborhood characteristics, and 11) Historical resources. According to $83-88 \%$ of respondents not performing on-site observation at all, the best method for improving and complementing the information regarding the climate is adding references related to the site. The best approach concerning the information of sensory perception is performing on-site observation (see figure 8).

It seems that in terms of almost all the attributes of the site, respondents tended to prioritize data obtained from lecturers, experts, or their colleagues than information that they found on the internet by themselves to improve the quantity and quality of the information. Climate is the attribute whose values can be found on the internet and regarded as sufficiently valid and reliable. Sensory perception is the attribute of the site that can only be understood well through an on-site survey, considering that this attribute relates to personal experience, which may differ across individuals.

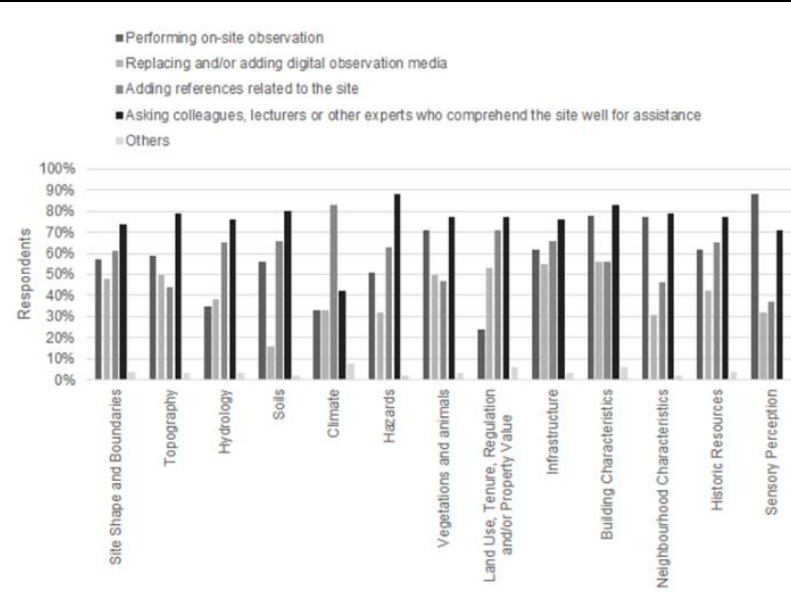

Source: Lukman and Sahid, 2021

Figure 8: Methods that are regarded as suitable for enhancing the level of identification of the site attributes

When a respondent not performing on-site observation answered that they could identify a specific attribute of the site adequately by depending on online or secondary data only, the respondent was asked whether the information concerning that particular attribute was helpful for the design process. Adequate information regarding all site attributes is valued as beneficial for the design process by $86-100 \%$ of respondents not performing on-site observation at all (see Figure 9). This implies that sufficient information concerning the site's attributes does support the design process.

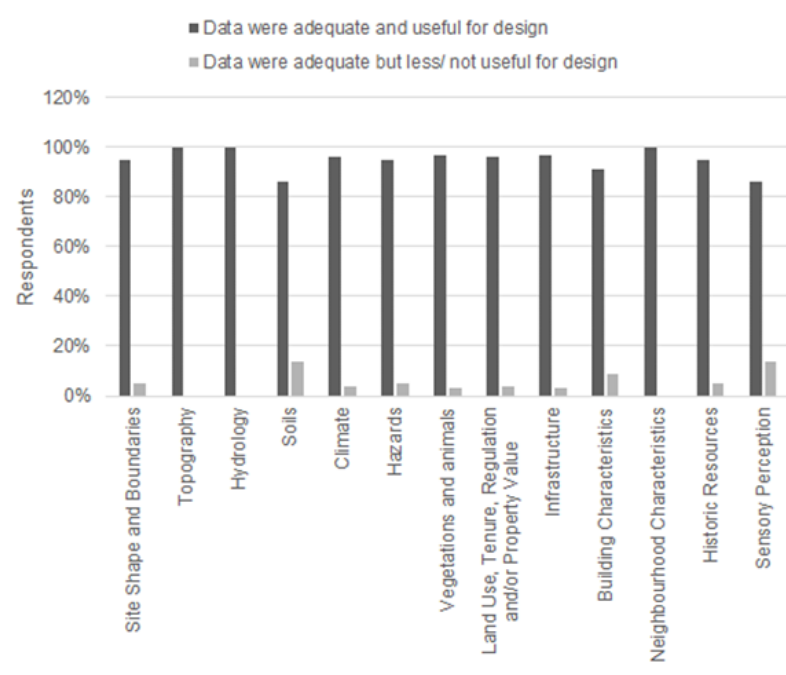

Source: Lukman and Sahid, 2021

Figure 9: The role of adequately identifiable site attributes in the design process 
Whether they performed on-site observation or not, all respondents were asked about what method(s) they regarded as best for site comprehension. Fifty-five respondents (54\%) consider that the best approach is performing on-site observation combined with collecting data from the internet or other people who are viewed as having more profound knowledge and understanding about the site (see Figure 10). Twenty-four respondents $(23,5 \%)$ consider that the best method is collecting data from both the internet and other people who are viewed as having more profound knowledge and understanding about the site (without conducting on-site observation) (see Figure 10). Twenty respondents $(19,5 \%)$ consider that the best method is performing on-site observation (without implementing other approaches) (see Figure 10). Only $1-2 \%$ of respondents regard collecting data from the internet or acquiring secondary data from other people as the best method (see Figure 10).

These results indicate that respondents, who are architecture students, think that obtaining comprehensive site data still needs offline observation at some stages. The results of this study are in accordance with referenced studies presenting that architecture students prefer the combination of on-site and online learning approaches in an architecture design studio to entirely online activities $[3,4,5]$.

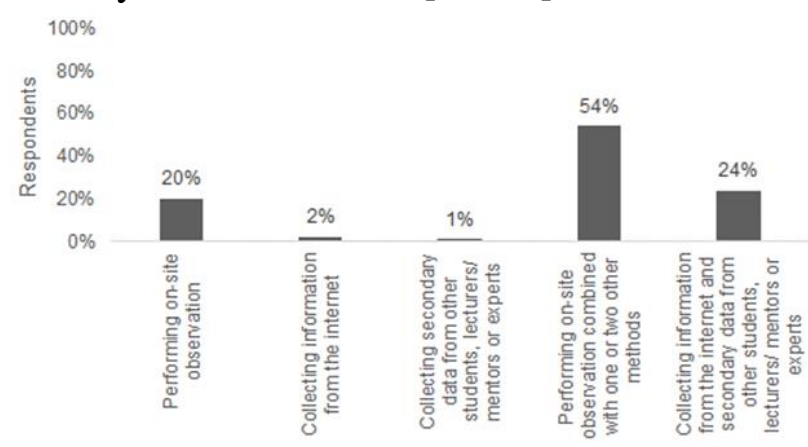

Source: Lukman and Sahid, 2021

Figure 10: Methods of data collection that are valued best for comprehending the site

\section{Conclusion}

Based on the results of this study, it can be concluded that when architecture students depend on mere audio or visual data collected from the internet or other people, without coming to the site, they tend to have partial comprehension regarding the site as context. Comprehending the site attributes is essential for supporting the design process, as implied by the respondents in this study. Therefore, contextual and responsive design output demands a comprehensive understanding of all site attributes. A thorough understanding of the site requires a combination of online and offline surveys encompassing a whole bodily experience, whether during the Covid-19 pandemic or afterward. It is recommended that the architecture design studio incorporate onsite observation concerning the site according to the health protocol so that the architecture students involved in the studio can better understand the site and eventually create more contextual and responsive designs.

The gap that this study intends to fill is the lack of supporting materials required for determining appropriate systems of architecture design studio, within and after the era of the Covid-19 pandemic, specifically in Indonesia. The results of this study might give a small contribution to filling the gap. Indeed, this study has limitations that should be managed in further research. The limitation of this study is that it involved only a small fraction of architecture students in the Undergraduate Program, Department of Architecture, Parahyangan Catholic University, Bandung, Indonesia. The results of this study need to be confirmed and complemented by further research addressing the implementation of an online architecture design studio that engages other students in this program or other departments of architecture in Indonesia. More comprehensive results can benefit the process of formulating more viable systems of the architecture design studio for the recent 
generation of architecture students and, eventually, the improvement of architecture education in Indonesia in the future.

\section{References}

[1] Al Maani, D., Alnusairat, S., \& Al-Jokhadar, A. (2021). Transforming learning for architecture: online design studio as the new norm for crises adaptation under COVID-19. Open House International.

[2] Ibrahim, A. F., Attia, A. S., Asma' M. B., \& Ali, H. H. (2021). Evaluation of the online teaching of architectural design and basic design courses case study: College of Architecture at JUST, Jordan. Ain Shams Engineering Journal, 12(2), 2345-2353.

[3] Iranmanesh, A., \& Onur, Z. (2021). Mandatory virtual design studio for all: exploring the transformations of architectural education amidst the global pandemic. International Journal of Art \& Design Education, 40(1), 251-267.

[4] Ceylan, S., Şahin, P., Seçmen, S., Somer, M. E., \& Süher, K. H. (2020). An evaluation of online architectural design studios during COVID-19 outbreak. ArchnetIJAR: International Journal of Architectural Research. [5] Salama, A. M., \& Crosbie, M. J. (2020). Educating architects in a post-pandemic world. Common Edge, 14(October), 6.
[6] Maturana, B., Salama, A. M., \& McInneny, A. (2021). Architecture, urbanism and health in a postpandemic virtual world. Archnet-IJAR: International Journal of Architectural Research.

[7] Paczkowski, P., Kim, M. H., Morvan, Y., Dorsey, J., Rushmeier, H. E., \& O'Sullivan, C. (2011). Insitu: sketching architectural designs in context. ACM Trans. Graph., 30(6), 182.

[8] Crotch, J., \& Deakin, I (2017). Site-Sense. Envisioning Architecture- Space/Time/ Meaning: EAEA 13th Biennial International Conference, 88.

[9] Asl, H. E., \& Mizban, P. (2016). Visual Perception in Architecture and Cinema, Similarities and Differences. International Journal of Engineering and Technology, 8(5), 345.

[10] Naz, A., Kopper, R., McMahan, R. P., \& Nadin, M. (2017, March). Emotional qualities of VR space. In 2017 IEEE virtual reality (VR) (pp. 3-11). IEEE.

[11] Shields, J. A., Gero, J. S., \& Yu, R (2017). Evaluating the veridicality of two-dimensional representations of three-dimensional architectural space through physiological response. The 10th EAAE/ARCC International Conference.

[12] LaGro Jr., J.A. (2008). Site Analysis: A Contextual Approach to Sustainable Land Planning and Site Design. New Jersey: John Wiley \& Sons, Inc. 
(This page is intentionally left blank) 